Images in...

\title{
Rescue surgery for a rare cause of pulmonary oedema
}

\author{
Sana Amraoui, ${ }^{1}$ Marion Marty, ${ }^{2}$ Michel Montaudon, ${ }^{3}$ Edouard Gerbaud ${ }^{1}$ \\ ${ }^{1}$ Soins Intensifs Cardiologiques - Plateau de Cardiologie Interventionnelle, CHU de Bordeaux, Hôpital Cardiologique du Haut Lévêque, Pessac, France \\ ${ }^{2}$ Laboratoire d'Anatomie Pathologique, Hôpital Cardiologique du Haut Lévêque, CHU de Bordeaux, Pessac, France \\ ${ }^{3}$ Unité d'Imagerie Thoracique et Cardiovasculaire, CHU de Bordeaux, Hôpital Cardiologique du Haut Lévêque, Pessac, France
}

Correspondence to Dr Edouard Gerbaud, edouard.gerbaud@chu-bordeaux.fr

\section{DESCRIPTION}

A 45-year-old man was referred to our department for acute respiratory distress. Upon arrival, chest x-ray showed an acute pulmonary oedema (figure 1) with a pulmonary tumour. Transthoracic echocardiography revealed a mass obstructing the majority of the left atrium and protruding into the left ventricle (figure 1). Eighteen years ago, the patient had been diagnosed with a grade II chondrosarcoma of the humerus, treated surgically. Three years ago, a local recurrence was again excised. This patient was recently awaiting a possible chemotherapy since the discovery of a pulmonary metastatic recurrence.

After intensive treatment with diuretics and non-invasive ventilation, a thoracic CT scan confirmed recurrence of the chondrosarcoma in the right inferior pulmonary lobe with complete thrombosis of the inferior pulmonary vein and invasion of the left atrium and ventricle (figure 1).

Surgical excision of the chondrosarcoma with pulmonary lobectomy and reconstruction of the left atrium was performed in emergency with success. Microscopic analysis revealed a well-differentiated chondrosarcoma, infiltrating the pulmonary parenchyma (figure 2). Tumour nodules were found in the vascular lumen (figure 2). Afterwards, the patient was treated with a new drug in evaluation for advanced chondrosarcoma, named Hedgehog pathway inhibitor vismodegib (GDC-0449).

The patient recovered well from surgery and was dismissed home 22 days after surgery. One month later, a
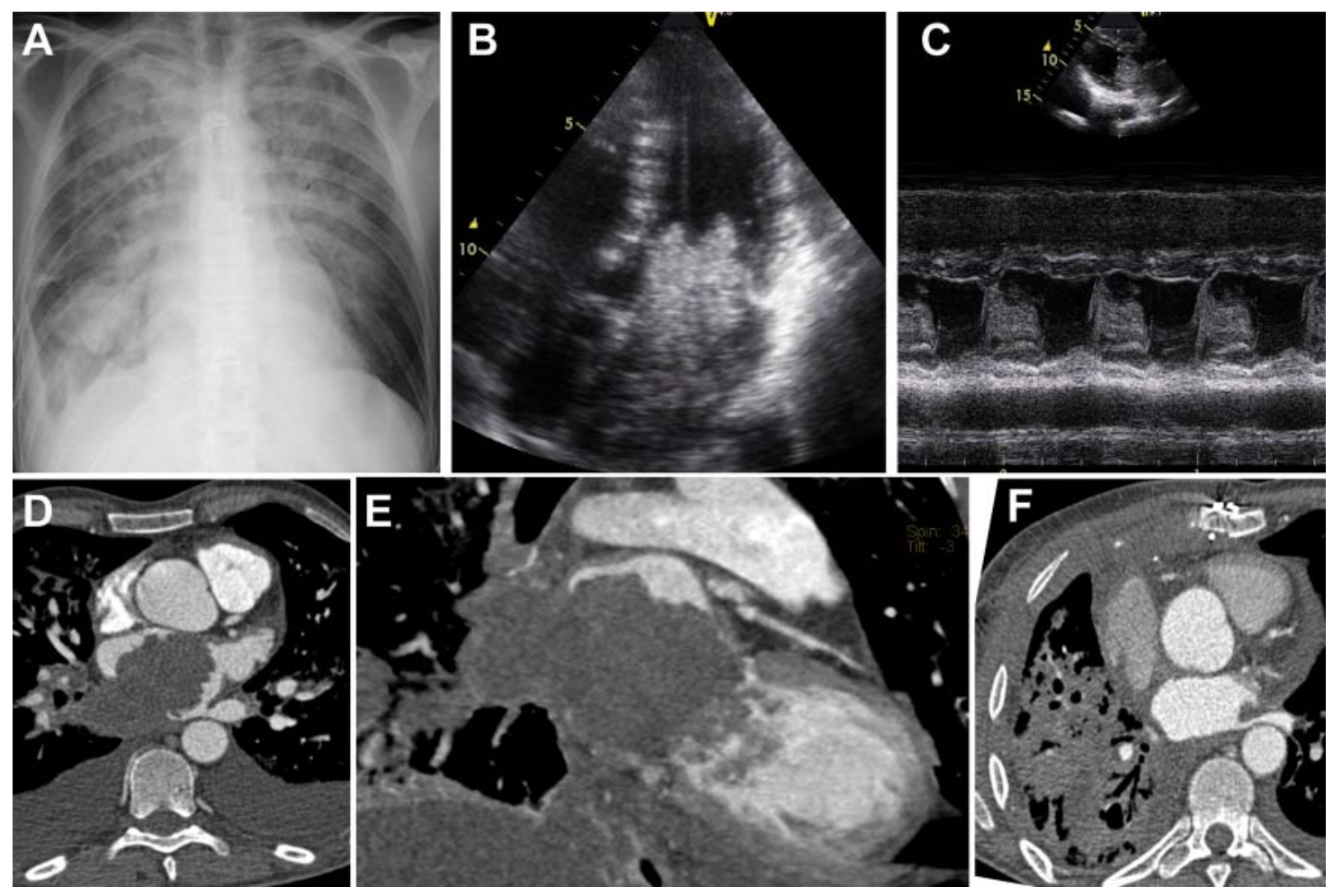

Figure 1 Chest $\mathrm{x}$-ray $(\mathrm{A})$ showing an acute pulmonary oedema with a pulmonary tumour. Apical two-dimensional four-chamber (B) echocardiogram showing a mass obstructing the majority of the left atrium and protruding into the left ventricle. Time-motion long axis parasternal (C) echocardiogram showing the mass protruding through the mitral valve. Thoracic CT axial (D) and two cavities multiplanar reconstruction (E) views confirmed recurrence of the chondrosarcoma in the right inferior pulmonary lobe with complete thrombosis of the inferior pulmonary vein and invasion of the left atrium and ventricle. One month later, a control CT scan (F) showed no cardiac recurrence of the chondrosarcoma. 


\section{BMJ Case Reports}

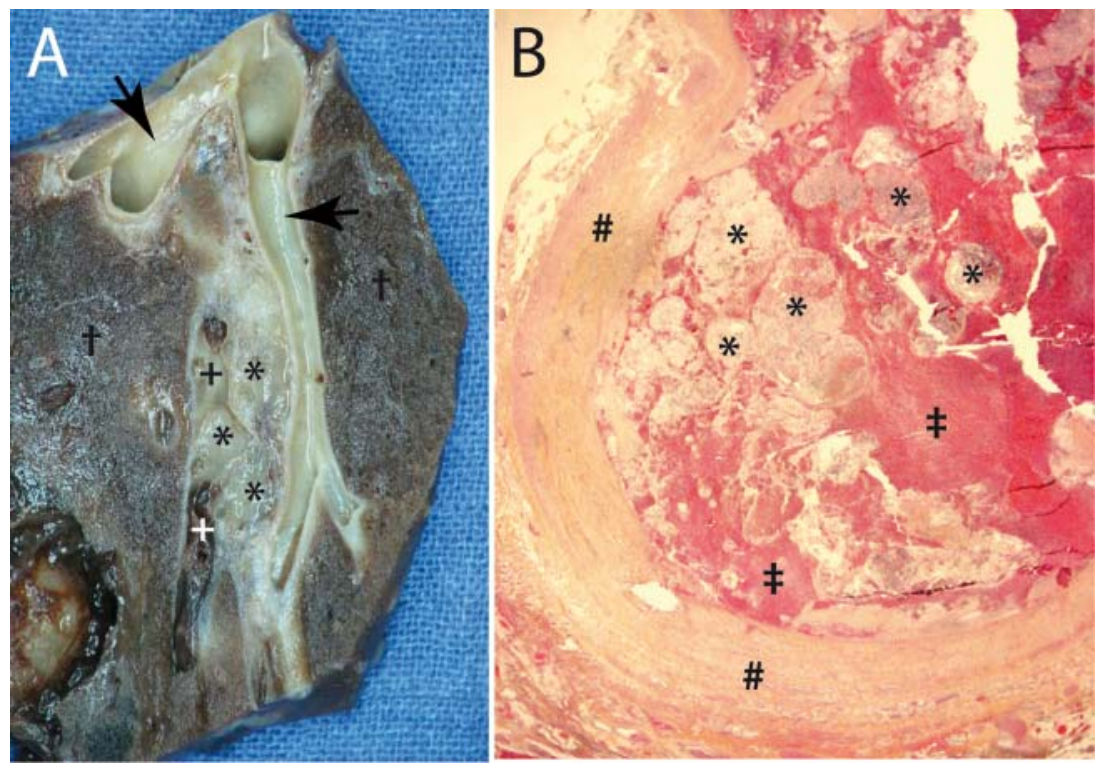

Figure 2 Gross examination — pulmonary parenchyma fixed with formalin $(A)$ showing tumoural material $\left({ }^{*}\right)$ within vascular lumen $(+)$ and infiltrating pulmonary parenchyma $(\dagger)$. Arrow indicates secondary bronchus. Histological examination—H\&E staining sections $(\times 10$ magnification) (B) showing intravascular emboli made of tumoural nodules characteristic of a chondrosarcoma. ( $¥$ ) indicates blood and (\#) vascular wall: pulmonary artery.

CT scan showed no cardiac recurrence of the chondrosarcoma (figure 1) with the patient in functional class New York Heart Association II
This case illustrates the life-saving benefit of extensive surgical excision in a rare case of chondrosarcoma metastasis obstructing the left cardiac chambers.

Competing interests None.

Patient consent Obtained.

This pdf has been created automatically from the final edited text and images.

Copyright 2012 BMJ Publishing Group. All rights reserved. For permission to reuse any of this content visit http://group.bmi.com/group/rights-licensing/permissions.

BMJ Case Report Fellows may re-use this article for personal use and teaching without any further permission.

Please cite this article as follows (you will need to access the article online to obtain the date of publication).

Amraoui S, Marty M, Montaudon M, Gerbaud E. Rescue surgery for a rare cause of pulmonary oedema. BMJ Case Reports 2012;10.1136/bcr-2012-006396, Published XXX

Become a Fellow of BMJ Case Reports today and you can:

- Submit as many cases as you like

- Enjoy fast sympathetic peer review and rapid publication of accepted articles

- Access all the published articles

- Re-use any of the published material for personal use and teaching without further permission

For information on Institutional Fellowships contact consortiasales@bmjgroup.com

Visit casereports.bmj.com for more articles like this and to become a Fellow 